\title{
Utilization of organosulphur compounds by axenic and mixed cultures of Rhodococcus rhodochrous IGTS8
}

\author{
Kevin J. Kayser, ${ }^{*}$ Barbara A. Bielaga-Jones, Kathy Jackowski, Opeoluwa Odusan \\ and JoHN J. KilBANE II \\ Institute of Gas Technology, 3424 South State Street, Chicago, Illinois 60616, USA
}

(Received 25 May 1993; revised 6 August 1993; accepted 23 August 1993)

\begin{abstract}
Growth assays reveal that Rhodococcus rhodochrous IGTS8 uses a wide range of organosulphur compounds as the sole source of sulphur, yet none of the compounds serve as carbon sources. Compounds that are utilized include thiophenes, sulphides, disulphides, mercaptans, sulphoxides, and sulphones. A convenient spectrophotometric assay (Gibbs assay), based on the chromogenic reaction of 2,6-dichloroquinone-4-chloroimide with aromatic hydroxyl groups, was developed and used in conjunction with GC/MS analyses to examine the kinetics of dibenzothiophene metabolism by axenic and mixed cell cultures of Rhodococcus rhodochrous IGTS8. The desulphurization trait is expressed at increasing levels during the exponential phase of growth and then declines in stationary-phase cells. Mixtures of streptomycin-resistant Rhodococcus rhodochrous IGTS8 and Enterobacter cloacae (an organism incapable of cleaving carbon-sulphur bonds in relevant test compounds) were prepared in ratios that varied over six orders of magnitude. Growth studies revealed that $E$. cloacae was able to gain access to sulphur liberated from organosulphur compounds by IGTS8; however, cell-to-cell contact appears to be required. These experiments also indicate that the desulphurization activity, on a per cell basis, is higher in mixed cultures than in axenic cultures.
\end{abstract}

\section{Introduction}

Sulphur dioxide released into the atmosphere from the combustion of crude oil and coal remains one of the most prominent and intractable issues of environmental concern as it is a major contributor to the generation of acid rain. Several technologies to prevent the release of sulphur compounds, from coal and crude oil, as atmospheric pollutants are currently being employed, but they remain expensive and, for the most part, they address only post-combustion sulphur removal. Microbial desulphurization may provide an improved technology for the pre-combustion removal of organic sulphur from fossil fuels.

Many reports have shown that several organisms possess the ability to remove the inorganic sulphur from coal (Hoffman et al., 1981; Isbister \& Kobylinski, 1985; Kargi \& Robinson, 1984; Silverman et al., 1963), but the organic sulphur content continues to be a tenacious

${ }^{*}$ Author for correspondence. Tel. +1 $3129493760 ;$ fax +1312949 3700.

Abbreviations: AED, atomic emission detector; DBT, dibenzothiophene; DBTSO, dibenzothiophene sulphoxide; $\mathrm{DBTSO}_{2}$, dibenzothiophene sulphone; 2-HBT, 2-hydroxybiphenyl. problem. Coal and crude oil contain many of the same organic sulphur structures, which include thiophenes, disulphides, thiols, sulphides, sulphones, mercaptans, and sulphoxides. The compound dibenzothiophene (DBT) has received most attention in recent biodesulphurization studies because it is an accepted model compound representative of thiophenic structures found in coal and petroleum. Several researchers have isolated organisms that possess the ability to use DBT as a carbon and a sulphur source for growth (Hou \& Laskin, 1976; Laborde \& Gibson, 1977; Malick, 1978; Monticello et al., 1985). Most of these organisms use the carbon-destructive pathway proposed by Kodama et al. (1973), in which degradation is initiated by the cleavage of carbon-carbon bonds. Some organisms have been shown to mineralize DBT completely (van Afferden et al., 1990). Recently, a Corynebacterium sp. (Omori et al., 1992) was reported to selectively cleave carbon-sulphur bonds. This culture used DBT as a sole sulphur source and produced 2-hydroxybiphenyl (2-HBP), as well as several hydroxynitrobiphenyls (from subsequent nitration).

The specific cleavage of carbon-sulphur bonds, rather than degradation/mineralization of organosulphur compounds, is preferred for a biodesulphurization 
process so that sulphur is removed but the carbon and calorific values remains intact. Rhodococcus rhodochrous IGTS8 is capable of the selective cleavage of carbonsulphur bonds in DBT, yielding 2-HBP as the sole detectable metabolite (Kilbane, 1989, 1990a). The removal of sulphur from coal and some model compounds by strain IGTS8 has also been demonstrated (Kilbane, 1989, 1990a,b, 1991; Kilbane \& Jackowski, 1992). In this paper, the range of organosulphur compounds metabolized by $R$. rhodochrous IGTS8 and the kinetics of metabolism of organosulphur compounds by axenic and mixed cell cultures of $R$. rhodochrous IGTS8 are reported.

\section{Methods}

Chemicals. All organic sulphur compounds were of the highest quality available and were obtained from Aldrich, Sigma, ICN Biomedicals, $\mathrm{K}$ and $\mathrm{K}$ Labs, or Eastman Kodak. Chemical compounds were filter-sterilized using an Acrodisc-Cr $0.2 \mu \mathrm{m}$ pore-size membrane filter (Gelman) or were sterilized by autoclaving.

Bacteria and growth conditions. Media (BSM) and growth conditions for $R$. rhodochrous IGTS8 (ATCC 53968) have been described previously (Kilbane, 1989, 1990a; Kilbane \& Jackowski, 1992). The majority of mixed cell culture experiments were mixtures of Enterobacter cloacae and $R$. rhodochrous IGTS8. The sulphur bioavailability assay (Kilbane, 1989, 1990 $b$; Kilbane \& Jackowski, 1992) was used to monitor the ability of $R$. rhodochrous IGTS 8 to use various organosulphur compounds as sole sources of sulphur. Growth tests using BSM lacking glycerol were performed to determine if $R$. rhodochrous IGTS8 could use organosulphur compounds as carbon sources (Kilbane, 1989, 1990a). General inhibition of the growth of $R$. rhodochrous IGTS8 in BSM/glycerol medium was assessed using $20 \mathrm{~mm}$-sulphate and $20 \mathrm{~mm}$ test compound. The specific ability to inhibit the desulphurization activity was assessed using $20 \mathrm{~mm}-\mathrm{DBT}$ and $20 \mathrm{~mm}$ test compound in growth experiments employing BSM/ glycerol media. The involvement of the desulphurization trait in the metabolism of a test compound was investigated by growing $R$. rhodochrous IGTS8 either with the test compound as a sole sulphur source or in the presence of the organosulphur test compound and sulphate, obtaining a washed-cell suspension, and quantifying the conversion of DBT to 2-HBP during a $1 \mathrm{~h}$ incubation using the standardized conditions of the Gibbs assay. Mixed culture experiments involved a two-chambered glass vessel separated by a dialysis membrane (Spectrum Spectra/Por membrane; molecular mass cut-off $1000 \mathrm{Da}$ ). Each chamber contained BSM supplemented with $20 \mathrm{mM}$ DBT. The vessel was incubated with shaking at $30^{\circ} \mathrm{C}$ and growth was monitored turbidimetrically in each chamber throughout the experiment. Culture supernatants employed in growth experiments were obtained from $R$. rhodochrous IGTS8 cultures, grown in BSM under sulphur-limiting conditions with DBT as the sole sulphur source, then centrifuged at $10000 \mathrm{~g}$ and/or filter-sterilized. Growth was monitored using a Klett-Summerson colorimeter equipped with a green filter; 100 Klett units corresponded to approximately $5 \times 10^{8}$ cells ml$^{-1}$. Cell density was also monitored by measuring the $\mathrm{OD}_{600}$. Colony-forming units were determined on nutrient agar (Difco). All growth and activity assays were performed in triplicate.

Analytical methods. Desulphurization activity was monitored using the Gibbs reagent (2,6-dichloroquinone-4-chloroimide, Sigma), GC/ MS, and by gas chromatography/atomic emission detector (GC)
AED). Gibbs reagent reacts with aromatic hydroxyl groups such as 2-HBP at a $\mathrm{pH}$ of 8.0 to form a blue-coloured complex that can be monitored spectrophotometrically at $610 \mathrm{~nm}$. To obtain the maximum accuracy using the Gibbs assay, the $\mathrm{pH}$ and time of incubation/colour development $(30 \mathrm{~min})$ must be precisely controlled. The linear range of the Gibbs assay is 0-1-10 p.p.m.

The Gibbs assay was used in two ways to monitor the desulphurization activity of bacterial cultures. The cumulative production of desulphurized product was determined by assaying aliquots removed at various times from cultures growing on an organosulphur compound such as DBT, that yields an hydroxylated aromatic compound as a desulphurization product. Alternatively, the desulphurization activity of cultures grown using any substrate, organosulphur or otherwise, was monitored by obtaining washed-cell suspensions in BSM, adjusting to a constant cell density of $\mathrm{OD}_{600}=1$, adding $20 \mathrm{~mm}$-DBT, incubating at $30{ }^{\circ} \mathrm{C}$ for $60 \mathrm{~min}$, and then using the Gibbs assay to determine the quantity of desulphurized product.

The Gibbs assay was done as follows: $5 \mathrm{ml}$ of microbial culture was placed in a $15 \mathrm{ml}$ test tube, the $\mathrm{pH}$ was adjusted to 8.0 with $10 \%(\mathrm{w} / \mathrm{v})$ $\mathrm{Na}_{2} \mathrm{CO}_{3}$, and then $50 \mu \mathrm{l}$ of Gibbs reagent $\left(10 \mathrm{mg} \mathrm{ml}^{-1}\right.$, ethanol solution) was added. The solution was allowed to incubate for $30 \mathrm{~min}$ to produce full colour development. The solution was then centrifuged to remove cells, and the absorbance of the supernatant was determined at $610 \mathrm{~nm}$ (Beckman DU-65) and converted to p.p.m. based on a 2-HBPgenerated standard curve. Samples for GC analysis were extracted from aqueous cell/DBT suspensions by liquid-liquid extraction using dichloromethane and analysed using a Perkin-Elmer 8320 with an AS$100 \mathrm{~B}$ autosampler. The column was $30 \mathrm{~m}$ long, $0.25 \mathrm{~mm}$ i.d., $0.25 \mu \mathrm{m}$ film, DB-5 type. The concentration range was $5-2000 \mu \mathrm{g} \mathrm{ml}^{-1}$ using the internal standard method of quantification.

\section{Results}

Growth on organosulphur compounds

The organosulphur compounds listed in Table 1 were tested as sole sulphur sources for the growth of $R$. rhodochrous IGTS8. GC/MS was used to examine the reaction products of several of the compounds. DBT, DBT sulphoxide (DBTSO), and DBT sulphone $\left(\mathrm{DBTSO}_{2}\right)$ were each uniquely converted to 2-HBP which was not further degraded or modified. $\mathrm{DBTSO}_{2}$ was observed as an intermediate in the metabolism of DBT. The compounds 2-hydroxy diphenyl sulphone and phenol were detected as metabolites of thianthrene.

All of the organosulphur compounds listed in Table 1 were examined for the ability to serve as the sole carbon source for growth of $R$. rhodochrous IGTS8 by using prolonged (weeks to months) incubations of $R$. rhodochrous IGTS8 using liquid and solid media. $R$. rhodochrous IGTS 8 was unable to use any of the organosulphur compounds listed in Table 1 as carbon sources.

Thianaphthalene, phenyl sulphoxide, 2-aminophenyl disulphide, and Thymol blue all appear to be generally inhibitory. The compounds tolyl disulphide, thionin, and sulphanilamide appear to be specific inhibitors of the desulphurization activity of $R$. rhodochrous IGTS8; they will not serve as sole sources of sulphur, although they will allow growth of $R$. rhodochrous IGTS8 when present 
Table 1. Metabolism of organosulphur compounds by $R$. rhodochrous strain IGTS8

\begin{tabular}{|c|c|}
\hline Compound & $\begin{array}{l}\text { Utilization } \\
\text { as sulphur } \\
\text { source }\end{array}$ \\
\hline DBT & + \\
\hline DBT sulphoxide & + \\
\hline DBT sulphone & + \\
\hline Thianthrene & + \\
\hline 1,4,7-Trithiacyclononane & + \\
\hline 1,5-Dithiacyclooctan-3-ol & + \\
\hline trans-1,2-Dithiane 4,5-diol & - \\
\hline 1,3-Dithiane & - \\
\hline 1,4-Dithiane & + \\
\hline 1,3-Propanethiol & - \\
\hline Thianaphthene & - \\
\hline Phenyl sulphoxide & + \\
\hline 2-Aminophenyl disulphide & + \\
\hline Thymol blue & + \\
\hline Tolyl disulphide & - \\
\hline Thionin & + \\
\hline Sulphanilamide & - \\
\hline Methionine & + \\
\hline Thiamine & + \\
\hline L-Cysteine hydrochloride hydrate & + \\
\hline Cystine & + \\
\hline Phenyl disulphide & - \\
\hline Thioxanthren-9-one & + \\
\hline Trithiane & + \\
\hline 2-Nitrophenyl disulphide & + \\
\hline 4-Aminophenyl disulphide & - \\
\hline 4-Nitrophenyl disulphide & + \\
\hline 3-Nitrophenyl disulphide & - \\
\hline Benzyl disulphide & + \\
\hline Biotin & - \\
\hline 2-Trimethylsilyl-1,3-dithiane & + \\
\hline Phenol red & + \\
\hline Methylene blue & + \\
\hline Thiazole yellow & + \\
\hline 2-Thiophene-carboxylic acid & - \\
\hline Thiazole & + \\
\hline Dimethysulphoxide & + \\
\hline
\end{tabular}

with sulphate but not when present with DBT. Some compounds that make excellent sulphur sources, such as methionine, cystine, thiamine and L-cysteine do not induce the expression of the desulphurization trait.

Growth studies also indicated that the expression of the desulphurization trait of $R$. rhodochrous IGTS8 was subject to both repression and induction. $R$. rhodochrous IGTS8 will not metabolize DBT in the presence of sulphate. The Gibbs assay was used to detect mutants of $R$. rhodochrous IGTS8 that will convert DBT to 2-HBP even in the presence of $20 \mathrm{~mm}$-sulphate. However, these mutant cultures did not express the desulphurization trait when grown with sulphate as the sole source of sulphur. They required the presence of a suitable organosulphur compound to induce the expression of the desulphurization trait.

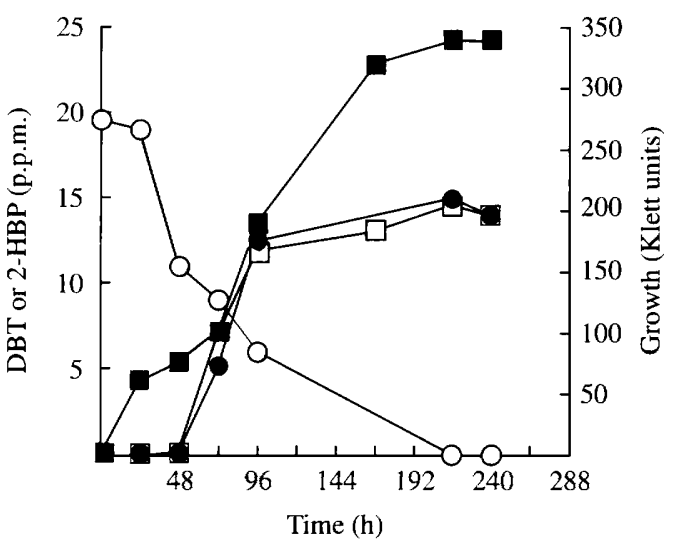

Fig. 1. R. rhodochrous IGTS8 growing-cell desulphurization of DBT. The DBT and 2-HBP data points are means of three uniform samples from each time point, with five separate determinations for each sample submitted to GC/MS analysis, totalling 15 samples for each DBT and 2-HBP GC/MS data point and three samples for each 2-HBP Gibbs assay data point. The average SD is 0.82 p.p.m. $\bigcirc$, DBT (p.p.m.); $\bullet$, 2-HBP measured by the Gibbs assay; $\square, 2-\mathrm{HBP}$ measured by GC/MS;

a, Growth.

The concentration of sulphur required for the growth of IGTS8 was investigated using sulphate, sulphite, DBT, DBTSO, and trithiane as sulphur sources at concentrations ranging from $0.005-5.0 \mathrm{~mm}$. About $0 \cdot 1 \mathrm{mM}$ of a sulphur source was required to achieve the maximum growth of $R$. rhodochrous IGTS8 in BSM, and the various sulphur sources mentioned above yielded similar results.

\section{Kinetics of desulphurization}

The expression of the desulphurization trait of $R$. rhodochrous IGTS8 in relation to the bacterial growth cycle was studied. $R$. rhodochrous IGTS8 was grown with DBT as sole source of sulphur, and the conversion/ accumulation of 2-HBP from DBT (or similar substrates) was monitored throughout the growth cycle. DBT was added at concentrations of 20 p.p.m. and the conversion of DBT to 2-HBP was monitored using both the Gibbs assay and GC/MS (Fig. 1). Growth and 2-HBP production ran parallel; DBT was converted to 2-HBP in near-stoichiometric amounts. Fig. 1 illustrates that the DBT concentration decreases by nearly half before significant quantities of 2-HBP are detected. This is thought to be partially due to the adherence of DBT and 2-HBP to bacterial cells (data not shown). A possible complicating factor in these experiments is that the end product 2-HBP, is somewhat inhibitory to cell growth and possibly to desulphurization activity, and its accumulation may bias the results. Moreover, because the cell concentration changes throughout the experi- 


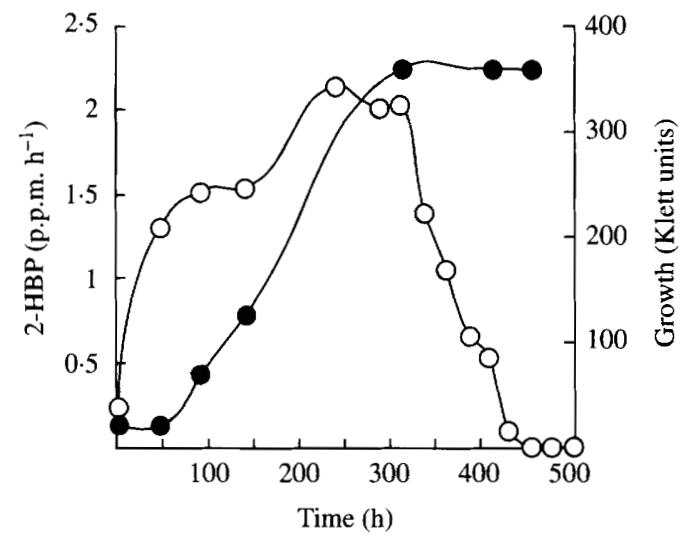

Fig. 2. R. rhodochrous IGTS8 resting-cell desulphurization of $\mathrm{DBTSO}_{2}$. Cell densities were adjusted to $\mathrm{OD}_{600}=1$ prior to being tested using the Gibbs assay. DBT was added for $1 \mathrm{~h}$. Each point is the mean of triplicate analyses. O, 2-HBP;, Growth cycle on DMSO.

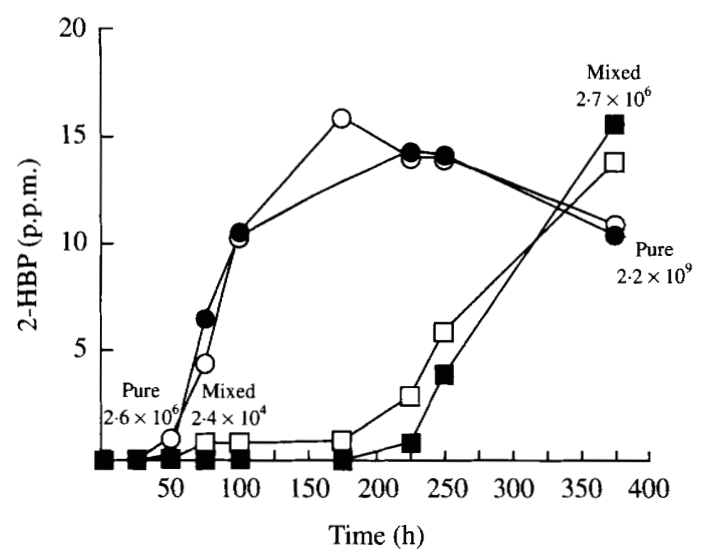

Fig. 3. The kinetics of production of 2-HBP by axenic and mixed cultures of $R$. rhodochrous IGTS8 grown with DBT as the sole sulphur source. The number of $R$. rhodochrous IGTS 8 cells ml ${ }^{-1}$ present in each culture at the beginning and at the end of the growth experiment is indicated. The SD of data points was based upon three uniform samples from each time point, with five separate determinations for each sample submitted to GC/MS analysis, totalling 15 samples for each GC/MS data point and three samples for each Gibbs assay data point. $\bigcirc, R$. rhodochrous IGTS8;,$R$. rhodochrous IGTS8; $\square, R$. rhodochrous IGTS8/mixed; $\mathbf{Q}, R$. rhodochrous IGTS $8 /$ mixed. $\bigcirc, \square$, Results obtained using the Gibbs assay; $\boldsymbol{\square}$, results obtained using GC/MS.

ment, changes in the specific activity are not readily apparent.

The data shown in Fig. 2 were obtained by growing $R$. rhodochrous IGTS8 with DMSO as the sole sulphur source and harvesting cells at various stages of growth, adjusting the cell density to $\mathrm{OD}_{600}=1 \cdot 0$, exposing the culture to $\mathrm{DBTSO}_{2}$ for $60 \mathrm{~min}$, and assaying using the Gibbs reagent. This method of analysis has the advantages of testing cells at identical cell densities and avoids the accumulation of high concentrations of the po- tentially inhibitory metabolite 2-HBP. The specific desulphurization activity of $R$. rhodochrous IGTS8 cells appeared to be relatively constant in early- to midexponential phase, peaked in late-exponential phase, and declined in stationary-phase cells (Fig. 2). A standard growth curve of $R$. rhodochrous IGTS8 grown on DMSO is included in Fig. 2 to show the phase of growth and cell densities at which the cell culture was harvested and assayed.

Tests using the Gibbs assay, as well as GC/MS analysis, indicate that the rate at which several compounds that have been proposed as pathway intermediates were metabolized by $R$. rhodochrous IGTS 8 cells during a $1 \mathrm{~h}$ incubation varied with the order DBTSO$_{2}>$ DBT $>$ DBTSO. The corresponding yields of 2-HBP produced from 20 p.p.m. of substrate, using the standard conditions of the Gibbs assay, were 5.0, 3.0, and 2.75 p.p.m., respectively. Prolonged incubation of $R$. rhodochrous IGTS8 cells for as long as $18 \mathrm{~h}$ yielded the same ranking of these three substrates with respect to rates and extent of metabolism.

\section{Axenic and mixed cell culture kinetics}

Fig. 3 shows quantitative cell-growth data, 2-HBP production data, and viable cell counts of pure $R$. rhodochrous IGTS8 cultures and mixed cultures of strain IGTS8 and E. cloacae. The $R$. rhodochrous IGTS8 strain used in these mixed culture experiments was resistant to $500 \mu \mathrm{g}$ streptomycin $\mathrm{ml}^{-1}$. The mixed cultures were plated onto nutrient agar with and without streptomycin. As indicated in Fig. 3, the cell density of $R$. rhodochrous IGTS8 in the pure culture was $2.6 \times 10^{6}$ cells ml $^{-1}$ after $40 \mathrm{~h}$ and $2.2 \times 10^{9}$ cells ml $^{-1}$ after $360 \mathrm{~h}$; whereas its concentration in the mixed culture was $2.4 \times 10^{4}$ cells $\mathrm{ml}^{-1}$ after $40 \mathrm{~h}$ and $2 \cdot 7 \times 10^{6}$ cells $\mathrm{ml}^{-1}$ after $360 \mathrm{~h}$. E. cloacae in pure culture was unable to grow under sulphur bioavailability assay conditions or convert DBT to detectable quantities of 2-HBP (data not shown). Even though these mixed cultures contained 200-fold fewer cells capable of metabolizing DBT, they eventually produced quantities of 2-HBP nearly identical to those of pure $R$. rhodochrous IGTS8 cultures.

Mixtures of $E$. cloacae and $R$. rhodochrous IGTS8 cultures were also prepared in various ratios. These mixed cultures were then used to inoculate test tubes containing BSM/glycerol medium with DBT serving as the sole source of sulphur. DBT and 2-HBP levels were monitored over a $15 \mathrm{~d}$ period. The streptomycin-resistant mutant of $R$, rhodochrous IGTS8 was used so that populations of E. cloacae and $R$. rhodochrous IGTS8 could be monitored. All of the cultures eventually grew to about the same density, although the growth rate was influenced by the ratio of E. cloacae to $R$. rhodochrous 
Table 2. R. rhodochrous IGTS8 and Enterobacter cloacae cultures prepared in various ratios

\begin{tabular}{|c|c|c|c|c|c|c|c|c|c|c|c|c|}
\hline \multirow{2}{*}{$\begin{array}{l}\text { Initial ratio of } \\
E \text {. cloacae to } \\
R \text {. rhodochrous } \\
\text { IGTS8 }\end{array}$} & \multicolumn{10}{|c|}{ Growth (Klett units) on day of incubation: } & \multirow{2}{*}{$\begin{array}{l}\text { Final ratio of } \\
E . \text { cloacae to } \\
R . \text { rhodochrous } \\
\text { IGTS8 }\end{array}$} & \multirow{2}{*}{$\begin{array}{c}\text { 2-HBP } \\
\text { (p.p.m.) } \\
\text { (Gibbs assay) }\end{array}$} \\
\hline & 1 & 2 & 3 & 6 & 7 & 8 & 9 & 10 & 13 & 15 & & \\
\hline IGTS8 & 33 & 47 & 50 & 46 & 260 & 415 & 520 & 525 & 560 & 590 & IGTS 8 & $11 \cdot 39$ \\
\hline $1: 1$ & 23 & 23 & 23 & 23 & 85 & 290 & 430 & 470 & 470 & 470 & $1 \cdot 3: 1$ & $9 \cdot 59$ \\
\hline $10: 1$ & 14 & 14 & 14 & 27 & 150 & 320 & 380 & 440 & 460 & 470 & $14 \cdot 5: 1$ & $9 \cdot 66$ \\
\hline $100: 1$ & 12 & 12 & 12 & 12 & 16 & 27 & 180 & 330 & 480 & 500 & $40 \cdot 3: 1$ & $8 \cdot 83$ \\
\hline $1000: 1$ & 13 & 13 & 13 & 13 & 17 & 24 & 122 & 300 & 460 & 500 & $96 \cdot 8: 1$ & $7 \cdot 82$ \\
\hline $10000: 1$ & 12 & 12 & 12 & 12 & 12 & 22 & 82 & 190 & 480 & 500 & $260: 1$ & 6.65 \\
\hline $100000: 1$ & 14 & 14 & 14 & 14 & 16 & 25 & 50 & 170 & 360 & 475 & $498: 1$ & $6 \cdot 33$ \\
\hline $1000000: 1$ & 13 & 13 & 13 & 13 & 17 & 22 & 33 & 88 & 425 & 470 & $1000: 1$ & $5 \cdot 17$ \\
\hline
\end{tabular}

IGTS8 and was more pronounced when the initial ratio was $\geqslant 100$ (Table 2). In cultures with initial ratios $<100$, the final ratio increased; whereas when the initial ratio was $>100$, the final ratio decreased. In some cases the final ratio decreased about 1000 -fold. Decreased final ratios indicate that $R$. rhodochrous IGTS8 grew proportionately more than $E$. cloacae, but this resulted in an overall decrease in the rate of growth.

Several growth experiments were performed with strain IGTS8 and E. cloacae cultures separated by a dialysis membrane. The dialysis membrane allowed the transfer of metabolites and nutrients but not microorganisms. $R$. rhodochrous IGTS8 grew well, whereas the $E$. cloacae culture failed to grow. In other experiments the supernatants of $R$. rhodochrous IGTS 8 cultures produced either by centrifugation or by filtration were examined for their ability to convert DBT to 2-HBP or to enable $E$. cloacae to metabolize DBT. No desulphurization was detected in experiments involving culture supernatants.

\section{Discussion}

$R$. rhodochrous IGTS8 has the ability to desulphurize/ utilize an extremely broad array of organosulphur compounds as sole sulphur sources including thiophenes, sulphides, disulphides, mercaptans, sulphoxides, and sulphones. Some points of the desulphurization of organosulphur compounds by $R$. rhodochrous IGTS8 can be concluded: carbon infrastructure is maintained during desulphurization; compounds that can serve as sulphur sources do not necessarily induce desulphurization activity; and some organosulphur compounds inhibit growth and/or desulphurization ability.

The metabolic pathway for the conversion of DBT to 2-HBP has been speculated to proceed by successive oxidative steps: DBT/DBTSO/DBTSO $/ \mathrm{DBTSO}_{3} / 2$ HBP and sulphite/sulphate (Omori et al., 1992; van
Afferden et al., 1990). The data obtained in this study were insufficient to either unequivocally confirm or deny the existence of this pathway. Alternative pathways for DBT biodegradation have been suggested for restingand growing-cell cultures of $R$. rhodochrous IGTS8, with the product of resting-cell metabolism being 2-HBP and that of growing cells being 2,2-dihydroxybiphenyl. Moreover two compounds, sultine (dibenz[c,e][1,2]oxathiin-6-oxide) and sultone (dibenz[c,e][1,2]-oxathiin6,6-dioxide), were isolated as intermediates in the DBT pathway (Gallagher et al., 1993). Although the examination of the metabolic pathway of DBT desulphurization was not the chief objective of this study, we have examined especially culture supernatants of exponentialphase, stationary-phase, and resting-cell cultures grown with, or exposed to, DBT for the presence of metabolites using GC/MS, GC/AED, and HPLC. We did not detect alternative metabolic pathways with cells examined in different phases of growth; we did not detect 2,2dihydroxybiphenyl as a metabolite but did observe $\mathrm{DBTSO}_{2}$ as an intermediate in the metabolism of DBT, and 2-hydroxydiphenyl sulphone was detected as an intermediate in the metabolism of thianthrene. DBT, DBTSO, and $\mathrm{DBTSO}_{2}$ were each converted uniquely to 2-HBP by $R$. rhodochrous IGTS8. The sulphur liberated from DBT (or other organosulphur compounds) was chiefly found associated with, or incorporated into the bacterial cells in experiments involving growing cells; however, experiments with resting-cell cultures indicate that sulphite is a reaction product (D. J. Monticello, personal communication). An analysis of headspace gases failed to detect hydrogen sulphide or any other sulphur-containing gas as a reaction product (data not shown).

Most of the compounds found in Table 1 can be used as sulphur sources but none can be used as carbon sources. Initially, $R$. rhodochrous IGTS8 appeared to be unable to use many of the compounds in Table 1 as 
sulphur sources; however, an adaptation/adjustment period prior to desulphurization/growth testing yielded the results shown. Adaptation consisted of subculturing $R$. rhodochrous IGTS8 cells from sulphur bioavailability assays that exhibited good growth into fresh BSM glycerol medium containing the organosulphur compounds of interest. The desulphurization of compounds such as 1,4,7-trithiacyclononane, 1,5-dithiacyclooctan-3ol, 1,3-dithiane, 1,4-dithiane, 1,3-propanethiol, and trans-1,2-dithiane-4,5-diol would be expected to yield products similar, if not identical, to ethanol or glycerol, if it is assumed that the cleavage of carbon-sulphur bonds results in the addition of a hydrogen atom or a hydroxyl group to the remaining hydrocarbon as has been shown for DBT, DBTSO, $\mathrm{DBTSO}_{2}$ and thianthrene. Because $R$. rhodochrous IGTS 8 can use glycerol and ethanol as carbon sources, it is unusual that no organosulphur compound has been found to serve simultaneously as a carbon as well as a sulphur source. The carbon infrastructure apparently remains intact, not only in desulphurization experiments with organosulphur compounds, but also in experiments with petroleum and coal; previous work showed that the calorific value was preserved in biodesulphurized petroleum and coal samples (Kilbane, 1991).

Perhaps the most interesting data in Table 1 are those of the compounds that appear to be either general or specific inhibitors of growth. Thianaphthalene, phenyl sulphoxide, 2-aminophenyl disulphide, and Thymol blue all appear to inhibit growth under all conditions. It is not clear if the inhibition was caused by the original compound or by some metabolite. The compounds tolyl disulphide, thionin, and sulphanilamide appear to be specific inhibitors of the desulphurization activity of $R$. rhodochrous IGTS8. They will not serve as sole sources of sulphur and will not allow growth when present with DBT but will allow growth of $R$. rhodochrous IGTS8 when present with sulphate. There is no obvious structure common to these three compounds or distinguishable from the other compounds tested that can be cited to explain the nature of the apparent specific inhibition of desulphurization activity.

The minimum sulphur requirement for growth of $R$. rhodochrous IGTS8 in BSM was about $0.1 \mathrm{mM}$. Similar results were observed for sulphate and a variety of organosulphur compounds. These data imply that strain IGTS8 does not metabolize DBT in excess of its requirement for growth. The results of experiments illustrated in Figs 1 and 2 show that the desulphurization trait is expressed at increasing levels during the exponential phase of growth and then declines in stationary phase.

We examined a variety of bacterial species in mixed cultures with $R$. rhodochrous strain IGTS8, including $E$. cloacae, E. agglomerans, Pseudomonas aeruginosa, and Klebsiella pneumoniae. All of the mixed culture experiments yielded similar results in that the quantity of DBT metabolized by mixed cultures and the total cell density were nearly equivalent to those of pure cultures of strain IGTS8, yet the relative abundance of strain IGTS8 cells in mixed cultures was several orders of magnitude lower than in pure cultures. Since $R$. rhodochrous IGTS8 is the only micro-organism present in the mixed cultures that is capable of utilizing DBT as a sulphur source (based on tests using pure cultures), while its relative abundance in mixed cultures is low, it appears that the per cell activity of $R$. rhodochrous IGTS8 cells is as much as 200 -fold higher in mixed cultures than in pure cultures. A better understanding of the physiological interaction with other bacterial cells in mixed culture may indicate means of increasing and/or prolonging the desulphurization activity of axenic cultures of $R$. rhodochrous IGTS8.

Sulphur liberated from DBT by $R$. rhodochrous IGTS8 can be used by other micro-organisms, but the fact that cell contact is required (as demonstrated by examining cultures separated by a dialysis membrane and by experiments with culture supernatants) indicates that a soluble sulphur form is not released into the medium under sulphur limiting conditions. Preliminary experiments indicate that desulphurization activity is found in the cell wall/membrane fraction of lysed strain IGTS8 cells (data not shown). These data imply a close association between strain IGTS8 and $E$. cloacae such that $E$. cloacae is capable of interrupting the uptake of sulphur by strain IGTS 8 cells in the mixed culture, 'stealing' sulphur liberated from DBT. This, plus the fact that polymeric organosulphur compounds far too large to be taken into the cytoplasm of bacterial cells are nonetheless successfully desulphurized by strain IGTS8 (Kilbane, 1991; Kilbane \& Jackowski, 1992), indicates that desulphurization occurs at the external surface of these cells.

The authors gratefully acknowledge the support of Energy Biosystems Corporation and the Illinois Clean Coal Institute for funding portions of this research.

\section{References}

van Afferden, M., Schacht, S., Klein, J. \& Truper, H. G. (1990). Degradation of dibenzothiophene by Brevibacterium sp. DO. Archives of Microbiology 153, 324-328.

Gallagher, J. R., Olson, E. S. \& Stanley, D. C. (1993). Microbial desulfurization of dibenzothiophene: a sulfur specific pathway. FEMS Microbiology Letters 107, 31-35.

Hoffman, M. R., Faust, B. C., Panda, F. A., Koo, H. H. \& Tsuchiya, H. M. (1981). Kinetics of the removal of iron pyrite from coal by microbial catalysts. Applied and Environmental Microbiology 42, 259-271.

Hou, C. T. \& LASKIN, A. I. (1976). Microbial conversion of dibenzothiphene. Developments in Industrial Microbiology 17, 351-362.

ISBISTER, J. D. \& KobYLINSKI, E. A. (1985). Microbial desulfurization 
of coal. In Processing and Utilization of High Sulfur Coals, vol. 9, Coal Science and Technology Series, pp. 627-641. Edited by Y. A. Attia. Amsterdam: Elsevier.

KaRGI, F. \& RoBinson, J. M. (1984). Microbial oxidation of dibenzothiophene by the thermophilic organism Sulfulobus acidocaldarius. Biotechnology and Bioengineering 26, 687-690.

Kilbane, J. J. (1989). Desulfurization of coal: the microbial solution. Trends in Biotechnology 7, 97-101.

Kilbane, J. J. (1990a). Biodesulfurization of coal. In Gas, Oil, and Coal Biotechnology, pp. 33-48. Edited by C. Akin \& J. Smith. Chicago: Institute of Gas Technology.

Kilbane, J. J. (1990b). Sulfur specific microbial metabolism of organosulfur compound resources. Conservation and Recycling 3, 69-79.

Kilbane, J. J. (1991). Microbial removal or organic sulfur from coal and petroleum. In Proceedings: 1991 Second International Biological Processing of Coal. Palo Alto, California: Electric Power Research Institute.

KiLBANE, J. J. \& JACKOWSKI, K. (1992). Biodesulfurization of water- soluble coal-derived material by Rhodococcus rhodochrous IGST8. Biotechnology and Bioengineering 40, 1107-1114.

Kodama, K., Umehara, K., Shimizu, K., Nakatani, S., Minoda, Y. \& YAMADA, K. (1973). Identification of microbial products from dibenzothiophene and its proposed oxidation pathway. Agricultural and Biological Chemistry 37, 45-50.

LABORDE, A. L. \& Gibson, D. T. (1977). Metabolism of dibenzothiphene by a Beijerinkia species. Applied and Environmental Microbiology 24, 783-790.

MALICK, K. A. (1978). Microbial removal of organic sulfur from crude oil and the environment: some new perspectives. Process Biochemistry 13, 10-12.

Monticello, D. J., Bakker, D. \& Finnerty, W. R. (1985). Plasmid mediated degradation of dibenzothiphene by Pseudomonas species. Applied and Environmental Microbiology 49, 756-760.

Omori, T., Mouna, L., SaIKI, Y.\& Kodama, T. (1992). Desulfurization of dibenzothiphene by Corynebacterium sp. strain SYI. Applied and Environmental Microbiology 58, 911-915.

Silverman, M. P., Rogoff, M. H. \& Wender, I. (1963). Removal of pyritic sulfur from coal by bacterial action. Fuel 42, 113-124. 